\title{
Redes Sociales como estrategia de comercialización en las empresas de manufactura automotriz
}

\section{Social Networks as a marketing strategies in automotive manufacturing companies}

RICO-CHAGOLLÁN, Mariana†, CHACÓN-OLIVARES, María del Carmen y RODRÍGUEZ CAMPOS, Juan Carlos y HERNÁNDEZ NEGRETE, Marcos Jesús Alejandro

Instituto Tecnológico Superior de Irapuato

ID $1^{\text {er }}$ Autor: Mariana, Rico-Chagollán / ORC ID: 0000-0001-6942-5902, Researcher ID Thomson: S-7659-2018, CVU CONACYT ID: 691659

ID $1^{\text {er }}$ Coautor: María Del Carmen, Chacón-Olivares / ORC ID: 0000-0002-3897-0235, Researcher ID Thomson: S-48062018, CVU CONACYT ID: 891518

ID $2{ }^{\text {do }}$ Coautor: Juan Carlos, Rodríguez-Campos / ORC ID: 0000-0002-8079-9654, Researcher ID Thomson: S-7721-2018, CVU CONACYT ID: 263767

ID $3^{\text {er }}$ Coautor: Marcos Jesús Alejandro, Hernández-Negrete

DOI: 10.35429/JIT.2019.18.6.9.16

Recibido: 10 de Enero, 2019; Aceptado 30 de Marzo, 2019

\begin{abstract}
Resumen
El presente trabajo aborda temas de actualidad, como lo son el uso de las Tecnologías de la Información y su incorporación en el plan de negocios de las organizaciones del sector automotriz. Por lo cual se realizó un análisis cuantitativo y de obtención de datos, que permitió establecer el aprovechamiento de las redes sociales como herramienta de comercialización, que ayude a mejorar los medios tradicionales de difusión de estrategias de comunicación efectiva en los mercados, además que impacte en el desarrollo de campañas digitales exitosas que permitan el posicionamiento de sus marcas y servicios. Por lo cual se detectó que del total de la muestra obtenida el $68 \%$ menciona que está dispuesto a utilizar las redes sociales para la difusión y promoción en campañas digitales, además fue contundente en un $95 \%$, el hecho de saber que prácticamente la totalidad de las encuestas arrojaron datos positivos, respecto al uso de redes sociales propias de cada organización, siendo las más usuales Facebook, Instagram y YouTube. Por lo tanto se determinó que el uso de las redes sociales por su cobertura, se consideran efectivas como una herramienta en la utilización de los procesos del diseño de comercialización.
\end{abstract}

Comercialización, Redes sociales, Tecnologías de la Información

\begin{abstract}
The present work addresses current issues, such as the use of Information Technology and its incorporation into the business plan of organizations in the automotive sector. Therefore, a quantitative analysis and data collection was carried out, which made it possible to establish the use of social networks as a marketing tool, to help improve the traditional means dissemination of effective communication strategies in the markets, as well as impacting on the development of successful digital campaigns that allow the positioning of their brands and services. Therefore, it was detected that $68 \%$ of the total sample shows that they are willing to use social networks for the dissemination and promotion of digital campaigns, and it was $95 \%$ the possitive answers in the surveys yielded positive data, regarding the use of social networks of each organization, being the most common Facebook, Instagram and YouTube. Therefore, it was determined that the use of social networks for their coverage is considered effective as a tool in the use of marketing design processes.
\end{abstract}

$\begin{aligned} & \text { Marketing, Social Networks, Information } \\ & \text { Technologies }\end{aligned}$

Citación: RICO-CHAGOLLÁN, Mariana, CHACÓN-OLIVARES, María del Carmen y RODRÍGUEZ CAMPOS, Juan Carlos y HERNÁNDEZ NEGRETE, Marcos Jesús Alejandro. Redes Sociales como estrategia de comercialización en las empresas de manufactura automotriz. Revista de Tecnologías de la Información. 2019. 6-18: 9-16

$\dagger$ Investigador contribuyendo como primer autor. 


\section{Introducción}

Sin duda alguna el crecimiento en la logística de información de las empresas en la actualidad ha generado que cada vez sea mayor el nivel de infraestructura digital necesario para almacenar datos, servicios y demás atributos de la cadena de valor en las organizaciones que permitan dar soporte a los requerimientos de cualquier proceso de certificación de calidad.

Ante esta revolución digital se le suma el hecho de tendencia actual en la comercialización de las estrategias de marketing que mediante aplicaciones de la web 2.0 y el cómputo en la nube, han generado un cambio en el diseño y distribución de las campañas publicitarias, permitiendo una cobertura mayor y generando como consecuencia la determinación de estrategias de diferenciación y segmentación de mercados. Sin embargo no todas las empresas cuentan con estos servicios, debido a temores comunes como el exponer información confidencial de la empresa, limitando de esta manera la utilización de las tecnologías de información.

En este proyecto se obtiene información directa por medio de la realización de cuestionarios a una muestra establecida con las características homogéneas que pueda representar a la población objetivo del estudio en las organizaciones con los que se podrá conocer cuáles son los principales paradigmas existentes en las empresas que les impiden integrarse a estas aplicaciones de las TI, siendo un caso de estudio debido a que de acuerdo a investigaciones realizadas por la plataforma "Hootsuite" dedicada a la gestión de redes sociales, se conoce el caso de empresas que mediante la integración de adecuadas campañas digitales han incrementado las ventas de algunos productos específicos, no solo generando mayor ingreso, sino adquiriendo una segmentación más definida de sus productos así como una interactividad con los usuarios de su perfil, dichos casos son de interés y según datos del análisis de tendencias en cuanto al uso de redes sociales en 2018 elaborado por Hootsuite nos encontramos con que plataformas como Facebook, Instagram y Twitter han incrementado su número de usuarios activos en un $35 \%$ respecto al año 2016.

\section{Justificación}

Actualmente muchas empresas no hacen uso o no cuentan con redes sociales propias de la organización ni tampoco con servicios externos de cómputo en la nube para llevar a cabo procesos de marketing digital, sino que más bien realizan sus procesos mediante la intranet de la organización, esto en gran medida se debe al poco aprovechamiento que se tiene sobre estas aplicaciones y al hecho de no obtener los beneficios de efectividad y productividad que estas herramientas generan en el entorno industrial. Desde el inicio de la web 2.0, se ha logrado la optimización de procesos y búsquedas, aumentando la participación e interacción del usuario dentro de la organización.

Con respecto al servicio de la nube, un ejemplo claro corresponde a las bibliotecas virtuales, ya que "Las bibliotecas han funcionado por mucho tiempo como depósitos de información, las cuales facilitan el acceso, recogiéndola y preservándola. Actualmente el entorno ha cambiado y las bibliotecas se enfrentan al reto de contener un creciente volumen de información digital, de ahí que son necesarias las tecnologías de información masiva" (Vázquez, 2015).

De tal manera que con el paso del tiempo el crecimiento en el almacenamiento de información y número de usuarios también ha generado el aumento en el tráfico de datos dentro de las empresas, lo que simultáneamente ha ocasionado que los métodos tradicionales de almacenamiento como usar computadoras propias de la empresa para guardar información sean insuficientes e incluso llegan a ser inseguros hoy en día para lograr almacenar toda la información necesaria, generando poca funcionalidad.

Por su parte las redes sociales son quizá el fenómeno más grande y que más usuarios ha unificado de todas las aplicaciones de la web, siendo promotoras de participación entre los usuarios y generando un impacto mediático en el cambio de las formas en que se realizaban las campañas publicitarias tradicionales, evolucionándolas hacia lo que vemos hoy en día en el contenido digital. 
El mundo corporativo no es una excepción a este fenómeno y cada vez más empresas hacen un uso intensivo de las TIC, lo cual les confiere la posibilidad de alcanzar altas eficiencias en la participación de usuarios. En concreto, aplicaciones como las redes sociales ofrecen a las firmas la posibilidad de reinventar y expandir muchas de sus actividades tradicionales como las de comunicación, marketing o venta de productos con el fin de llegar mejor a los consumidores.

\section{Problema}

Hoy en día el uso de las redes sociales utilizadas como canales de comunicación y difusión de campañas publicitarias es más frecuente en empresas de todos los tamaños, ya que "Según la International Data Corporation, el comercio electrónico ha triplicado sus ventas en los últimos seis años." (Banco Bilbao Vizcaya Argentaria, S.A., 2018, párr. 1). Sin embargo, la gestión de estas aplicaciones, lejos de estar orientadas hacia su función principal que es analizar el comportamiento del cliente respecto a los productos y servicios que se ofrecen, están frecuentemente direccionadas hacia el fracaso en su aprovechamiento ya que en la mayoría de las empresas aunque cuentan con al menos una red social, es muy poco el uso que se les da, es por eso que el principal problema detectado corresponde a la desconfianza existente en el uso de la TIC como herramienta que les permita generar información que posteriormente será empleada por las redes sociales para generar campañas publicitarias en la web, conociendo de esta manera el comportamiento del consumidor. Esto ante el posible desconocimiento del potencial de uso que estas aplicaciones tienen para el desempeño de las campañas publicitarias mediante medios digitales.

\section{Hipótesis}

La utilización de las TIC's para generar información que en colaboración con las redes sociales puedan crear campañas digitales, de marketing que les permitan llegar a posibles clientes e incluso segmentar de una manera más eficaz su mercado.

\section{Objetivos}

\section{Objetivo General}

Analizar la integración de las redes sociales como herramienta de comercialización en las organizaciones del sector automotriz.

\section{Objetivos Específicos}

- Inquirir el proceso para el desarrollo de campañas de comercialización en. empresas automotrices

- Identificar el aprovechamiento de la incorporación de las TICS como estrategias de comercialización en empresas del sector automotriz.

Aplicación de encuestas en un grupo de empresas del sector automotriz para identificar el uso actual de las TIC en las organizaciones.

\section{Marco Teórico}

En esta investigación se hará mención a diferentes términos como lo son:

Interconectividad: Es uno de los elementos claves de las redes sociales. Seres en puntos diversos y distantes del planeta están conectados con comunidades de intereses afines, sin que se conozcan previamente y sin que hayan tenido que hacer importantes inversiones previas de movilización para reunirse $\mathrm{y}$ conocerse. (Pérez, 2011)

- Web 2.0: Es un término que apareció en el año 2003 dando nombre a una nueva era que se conformó a través de distintas aplicaciones en internet. Con este nombre se pone fin a una primera etapa en internet de la web, donde el internauta era únicamente el sujeto pasivo que obtenía la información o la exponía, sin que los internautas tuvieran posibilidad de interactuar. (Pérez, 2011)

- Redes sociales: son aplicaciones de la Web 2.0 que identifican a sitios tan populares y de uso diario como Facebook, Twitter, MySpace o LinkedIn. Éstos permiten a sus usuarios construir un perfil público o semi-público en sus plataformas, tener una lista de usuarios o conocidos con los que tienen conexión y ver las listas de conexiones de otros usuarios en el sistema (Boyd y Ellison 2010).

RICO-CHAGOLLÁN, Mariana, CHACÓN-OLIVARES, María del Carmen y RODRÍGUEZ CAMPOS, Juan Carlos y HERNÁNDEZ NEGRETE, Marcos Jesús Alejandro. Redes Sociales como estrategia de comercialización en las empresas de manufactura automotriz. Revista de Tecnologías de la Información. 2019 
Además, ofrecen la posibilidad de dejar mensajes públicos en los perfiles de los amigos, al igual que enviar mensajes privados de una forma similar al correo electrónico. (Ayala, 2014)

- Tecnología de la Información: Las tecnologías de la información se pueden entender como el conjunto de procesos y productos relacionados con el almacenamiento, procesamiento, protección, monitoreo, recuperación y transmisión digitalizada de la información tanto a nivel electrónico como óptico. (Saavedra \& Tapia, 2013)

Cómputo en la nube: Es un término general que se refiere a la prestación de servicios alojados a través de Internet. Se trata de una tecnología avanzada que hace que todos los archivos, programas e información estén almacenados en Internet, como en una "nube", siendo completamente irrelevante las capacidades de almacenaje de los ordenadores instalados por el cliente y prescindiendo así de los discos duros. (Miralles, 2010)

Teletrabajo: El teletrabajo es una forma flexible de organización del trabajo que consiste en el desempeño de la actividad profesional sin la presencia física del trabajador en la organización de manera física. (Fuentes, 2011)

Intranet: La intranet es una de las tecnologías más poderosas que pueden utilizarse en una organización, si se aplica de forma adecuada. Su diseño e implementación, en función de los objetivos organizacionales, provee a la institución de una herramienta fundamental para la gestión de la información que tributa a la gestión del conocimiento, y para la comunicación interna, que permite mejorar y agilizar la dinámica organizacional. (Rodríguez, 2017)

\section{Tipo de Investigación}

Esta investigación hará uso de una investigación descriptiva, documental, de campo y exploratoria, partiendo del objeto de estudio con la finalidad de conocer e interpretar mejor su concepto. Como lo menciona Sampieri, (2014), la investigación exploratoria ofrece identificar un análisis general del problema, identificando conceptos claves para el estudio del caso específico.

\section{Métodos Teóricos}

Actualmente México vive un crecimiento importante en cuanto a usuarios conectados a internet con acceso a teléfonos inteligentes e interconectividad mediante redes sociales.

"Con datos del $14^{\circ}$ Estudio sobre los Hábitos de los Usuarios de Internet en México 2018, la tasa de crecimiento promedio anual del número de usuarios de internet pasó de 51.2 millones de usuarios en 2013 a 79.1 millones de usuarios en 2018” (Martínez, 2018).

Tal y como se observa en la Figura 1; "Ochenta y tres millones de usuarios en México mensualmente presentan actividad en Facebook, de este número, más del $90 \%$ accede desde un móvil, siendo los perfiles de mujeres los que presentan un mayor número, así como usuarios entre los 18 y 34 años (edad declarada en el perfil) los que hacen mayor uso de la red social." (Martínez, 2018)

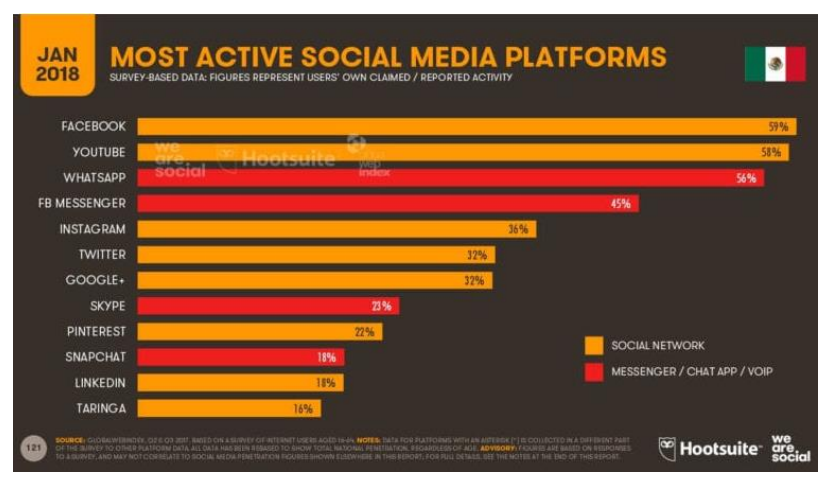

Figura 1 Actividad de redes sociales en México 2018 Fuente: Obtenido de (Copp \& Adame, 2018)

Por su parte una de las primeras observaciones fueron las campañas de Marketing digital desarrolladas por empresas Chevrolet y Ford, las cuales según (NEXU, 2018), "Encontraron en las redes sociales la herramienta necesaria para saber lo que el cliente quiere sin la necesidad de gastar mucho dinero en encuestas personalizadas a cada uno". Y es que realmente la utilización que estas empresas le dan a las redes sociales se caracteriza por hacer uso de técnicas de marketing digital a fin de entender a su mercado.

Ante esto surgió la duda respecto a si las redes sociales estaban destinadas unicamente a empresas grandes y previamente establecidas en el mercado, o si solo podían ser empleadas por empresas de venta directa del automóvil y dejando fuera a las empresas dedicadas a contrucción de autopartes.

RICO-CHAGOLLÁN, Mariana, CHACÓN-OLIVARES, María del Carmen y RODRÍGUEZ CAMPOS, Juan Carlos y HERNÁNDEZ NEGRETE, Marcos Jesús Alejandro. Redes Sociales como estrategia de comercialización en las empresas de manufactura automotriz. Revista de Tecnologías de la Información. 2019 
Las empresas de autopartes y manufactura a menudo no cuentan con una segmentación de mercado propia. Esto debido a que su meta no es distribuir a personas físicas, sino la de lograr distribuir a empresas que ensamblen el vehículo. Ante ello muchas veces se tiene la creencia de que no es necesario contar con una estrategia de marketing, campaña digital o medio de difusión "red social". Sin embargo este pensamiento es completamente erróneo. Empresas como Pirelli o Continental, dedicadas a la fabricación de neumáticos cuentan con una fuerte presencia en redes sociales pese a que su mayor fuerza de ventas son precisamente ensambladoras.

Sin embargo el uso que le dan a las redes sociales va más allá de compartir información técnica de la empresa. Su uso se concentra en desarrollar contenido multimedia a fin de interactuar con los usuarios de las redes sociales, con la finalidad de identificar si los modelos expuestos en los videos o imágenes son del agrado del público y poder generar mejoras a dichos modelos. Además de esto se crea presencia en algunas de las redes mas empleadas como Facebook, Intagram o Twiter, lo cual es un mediador de éxito, ya que genera en el usuario un sentido de identidad con la marca lo que a largo plazo también genera en el consumidor final un detonante, como por ejemplo; cuando este usuario decida cambiar de neumáticos en su auto elija alguna marca que él recuerde de estos medios donde previamente recibió información de la marca.

Guanajuato actualmente es uno de los clúster automotrices más grandes del país. Cerca del municipio de Irapuato se encuentran algunos parques y ciudades industriales que concentran a la mayoría de las empresas automotrices del Estado. Por ello se decidió aplicar encuestas a un grupo específico, el cual actualmente se encuentra laborando en áreas administrativas de estas empresas, esto con el fin de identificar si en estas empresas se hace uso de las redes sociales y el cómputo en la nube, con la finalidad de identificar de que manera utilizan las empresas estas herramientas.

Lo primero que se realizó fue identificar si los usuarios concoian el termino computo en la nube, lo cual se muestra en la figura 2 , en donde se confirma que en ocasiones el descnocimiento conlleva a una limitante del uso adecuado de la herramienta.

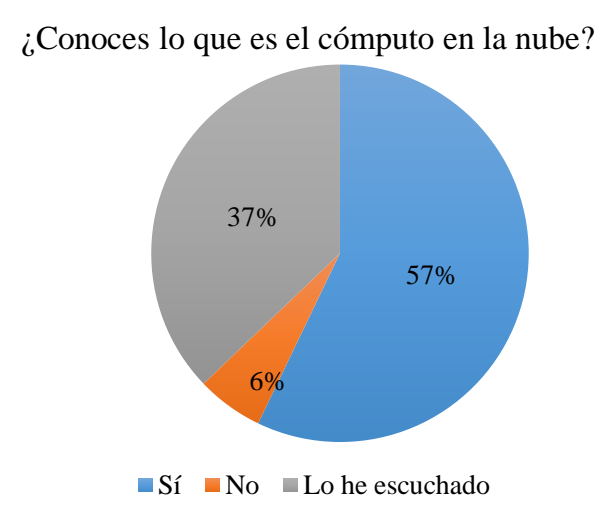

Figura 2 Conocimiento respecto a herramienta de cómputo en la nube

Fuente: Elaboración Propia

Luego de investigar lo que para los usuarios entrevistados era una clara definición del cómputo en la nube nos encontramos con algunas respuestas tales como:

"Es una base de datos propia de la organización para tomar decisiones estratégicas y tener un orden y control de la misma."

"Es un programa que permite guardar y analizar un gran número de datos facilitando más decisiones y la organización de una empresa."

\section{"Conjunto de datos confidenciales de la organización."}

Lo cuál permite tener una perspectiva más clara respecto a lo que se conoce del cómputo en la nube dado que los resultados de las encuestas arrojan que los usuarios identifican esta herramienta mas como un medio masivo de información referente a la organización y no tanto como un potencial aliado de los motores de busqueda y segmentación para las redes sociales.

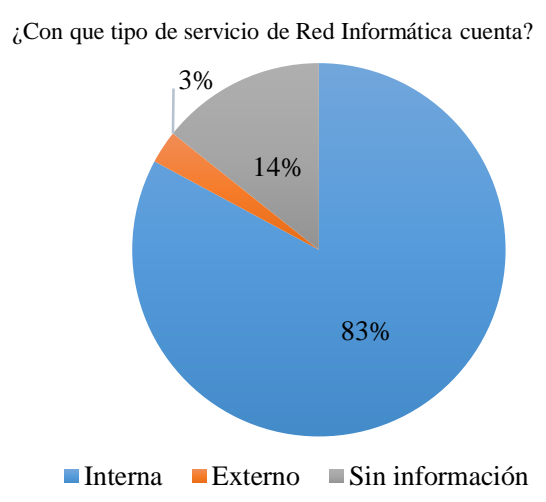

Figura 3 Información respecto al tipo de servicio empleado en las organizaciones

Fuente: Elaboración Propia

RICO-CHAGOLLÁN, Mariana, CHACÓN-OLIVARES, María del Carmen y RODRÍGUEZ CAMPOS, Juan Carlos y HERNÁNDEZ NEGRETE, Marcos Jesús Alejandro. Redes Sociales como estrategia de comercialización en las empresas de manufactura automotriz. Revista de Tecnologías de la Información. 2019 
La figura 3 muestra como en su mayoría las empresas poseen servicios de red propios dentro de la organización, lo que permite a los responsables proteger la información confidencial de la empresa. En la mayoría de los casos, esto se hace debido al riesgo potencial existente al exponer datos confidenciales con un tercero, lo cual es totalmente valido, sin embargo una de las principales desventajas que esto proporciona es la lentitud ya que en la mayoría de los casos los servidores internos de la organización no se encuentran en las plantas de manufactura, sino que se encuentran en la empresa matriz de estas, encriptados y sumamente protegidos. Lo que si bien representa una ventaja de seguridad, también disminuye considerablemente la velocidad de consulta de información en tiempo real, lo que en casos como el Teletrabajo o "home office" dificulta estas labores.

Una vez comprendida esta parte respecto a sí se tiene conocimiento de lo que es el cómputo en la nube se abordó otro tema de interés como lo es las redes sociales, vistas de una perspectiva empresarial orientada al marketing digital.

\section{¿Se cuenta con al menos una red social en la organización?}

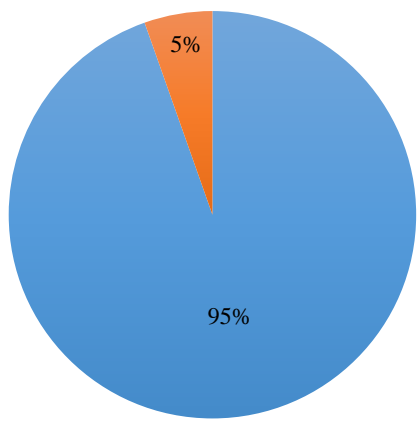

$\square$ Sí $\square$ No

Figura 4 Uso activo de redes sociales en las organizaciones

Fuente: Elaboración Propia

La figura 4 muestra como de la muestra establecida se obtiene que un $95 \%$ de las empresas cuenta con redes sociales propias de cada organización, siendo las más usuales Facebook, Instagram y YouTube. Sin embargo al consultar e indagar respecto al uso actual que estas empresas le dan a sus redes sociales se obtuvo resultados en donde la mayoría de los casos es nulo su contenido digital e incluso únicamente se emplean como mediadores de información.
Entonces se pretendió entender por qué pasaba esto preguntando a los encuestados respecto a los beneficios que a su parecer traía contar con redes sociales en la organización. Respuestas cómo; "Más información sobre la empresa, más solicitudes de empleados, etc.", "Adentrarse a la comunicación por medio de redes y expandir el público objetivo además de ser un medio de información" o "Como Recursos Humanos, tiene el beneficio de publicar las vacantes". Nos dejaron claro que la educación digital respecto al potencial uso de redes sociales era bajo.

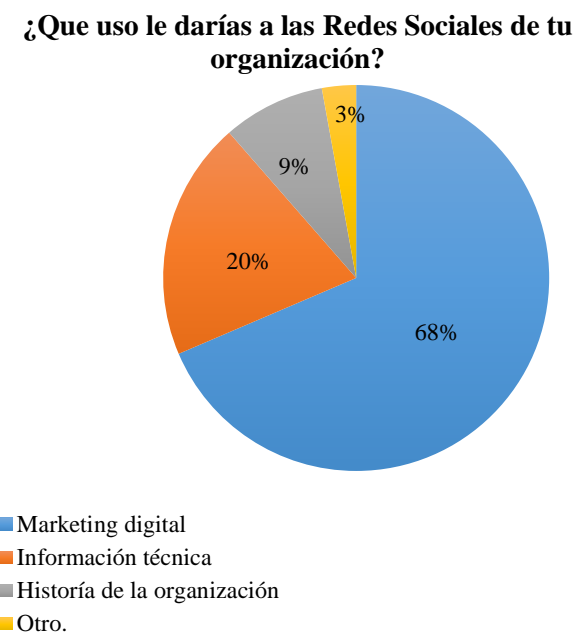

Figura 5 Finalidad de redes sociales en las organizaciones.

Fuente: Elaboración Propia

De tal manera que la figura 5 nos deja claro que en un $68 \%$ de las veces el uso que se le pretende dar a las redes sociales es completamente orientado hacia el marketing digital, mediante la creación de las llamadas campañas digitales.

Pero, ¿Qué tanto conocimiento real se tiene en las organizaciones de lo que son las campañas digitales? Al encuestar a los usuarios se observó que las palabras que más se repetían en sus respuestas eran; "Pagar dinero", "Darse a conocer" y "Publicar mucho". Lo cual inmediatamente nos demostró que no se tiene un sólido concepto del potencial que estas brindan al negocio.

Una vez realizada una investigación respecto a cómo operan los servidores digitales y bases de datos de redes como Facebook o Instagram. Se observa que los datos obtenidos varían respecto al giro comercial que se le pretenda dar al perfil, este giro es determinante para lograr llegar al público meta de las organizaciones.

RICO-CHAGOLLÁN, Mariana, CHACÓN-OLIVARES, María del Carmen y RODRÍGUEZ CAMPOS, Juan Carlos y HERNÁNDEZ NEGRETE, Marcos Jesús Alejandro. Redes Sociales como estrategia de comercialización en las empresas de manufactura automotriz. Revista de Tecnologías de la Información. 2019 
Empresas que venden autos ya terminados tienen un público meta más definido y por lo mismo se dedican a crear contenido digital para vender. Sin embargo empresas orientadas hacia la manufactura de autopartes desarrollan contenido orientado hacia conocer el concepto o idealismo del consumidor, gustos e intereses. Así como saber si son de estilos clásicos del diseño tradicional de la marca o atrevidos con conceptos más actuales y de tendencia. Como por ejemplo empresas de neumáticos, que en sus anuncios publicitarios desarrollan imágenes frescas en carretera probando la resistencia de los neumáticos con coches deportivos y actores jóvenes, son diferentes a imágenes cálidas de actores simulando una familia probando la resistencia de las llantas en una camioneta familiar con toda la familia en un paseo camino a la escuela o trabajo.

Esto es debido a que a pesar de que dos empresas se dediquen a fabricar neumáticos, una puede enfocar su venta a autos tipo deportivos, mientras que la otra estará orientada a vender neumáticos para autos familiares, a pesar de esto realizan anuncios digitales para sacar modelos o incluso algunas veces ni siquiera se menciona al neumático simplemente quieren entender al usuario final para a partir de ahí direccionar sus procesos con un sentido más definido logrando con esto un mejor posicionamiento de marca y sobretodo reconocimiento entre el público a pesar de que directamente no sean clientes.

Pero, ¿Por qué relacionar estos dos conceptos? Pues los motores de las redes sociales funcionan con algoritmos predefinidos por los datos que los usuarios introduzcan, el tipo de publicación y los canales de distribución.

En ocasiones las empresas crean cuentas $\mathrm{y}$ al no conocer cómo funcionan las redes terminan por publicar contenido sin un rango de edad definido, sin un tipo de público definido y sobre todo, sin una idea clara de lo que se pretende lograr.

Al ser una fuente de almacenamiento masivo de información, se cuenta con toda la información necesaria para nutrir los motores de búsqueda en las redes sociales, sin llegar a exponer información confidencial de la organización, únicamente recopilando aquellos datos que sean de interés para definir la estrategia comercial que se empleará.
Finalmente uno de los últimos datos que se pretendía conocer era si existía aún la creencia limitante de que las redes sociales eran únicamente para empresas de venta directa con el consumidor o "gigantes de la comercialización". Ante ello se formuló la pregunta que pretendía indagar este hecho, afortunadamente se obtuvieron resultados positivos ya que prácticamente la totalidad de los encuestados señaló estar de acuerdo con que las redes sociales son una oportunidad de crear campañas más exitosas, a pesar de que actualmente por lo observado no se esté realizando de manera tan efectiva como se pretende o simplemente se sigan realizando estas prácticas por los llamados, "medios tradicionales de difusión".

\section{Conclusión}

Luego de realizar la investigación teórica respecto al uso de tendencia que actualmente se les da a las campañas digitales mediante medios como Facebook o Instagram, se generaron dudas respecto al uso que las empresas de manufactura le daban a las mismas, para ello se realizó la investigación de campo tomando como base los resultados estudiados en la investigación teórica para nutrir el cuestionario aplicado a los usuarios encuestados.

Como resultado se obtuvieron datos contundentes que demostraron el uso poco eficaz que se le da actualmente a las redes sociales por parte de estas organizaciones. En la mayoría de los casos estudiados las organizaciones contaban con perfiles en Facebook o Instagram e incluso YouTube, sin embargo el uso que le dan actualmente es meramente de información técnica y en algunos casos ni siquiera tienen ningún tipo de información, es meramente la página estática.

En otros casos donde nos encontramos con empresas que si hacen uso de estos medios, se identificó en los usuarios una idea más clara de lo que son las campañas digitales y claro, esto debido a que en los perfiles sociales de la organización se cuenta con contenido multimedia no solo de carácter informativo de la organización, sino también de carácter comercial. 
Esto no solo ayuda a la imagen de la organización sino que los usuarios encuestados incluso tienen una idea más sólida y firme de lo que la empresa realiza dentro y fuera del núcleo central de los trabajadores, sino también factores externos como publicidad, acciones sociales, etc. De tal manera que el uso eficaz de las redes sociales en las organizaciones no solo genera un aumento en la presencia de la marca sino que además permite el desarrollo de estrategias de comercialización digital, ya que su uso e implementación en las organizaciones permite comunicar de mejor manera el sentido de la marca, crea lazos con los usuarios, expone fielmente el objetivo de la organización y finalmente agiliza los procesos de segmentación del mercado para desarrollar campañas publicitarias más eficaces y que logren impactar de forma positiva a un público previamente establecido en los motores de búsqueda, alimentado por la misma información que la empresa ya tiene.

\section{Referencias}

AMDA. (2018). AMDA. Obtenido de https://www.amda.mx/estudio-sobre-elpotencial-de-mercado-de-vehiculoscomerciales-y-pesados/

Ayala, T. (2014). Redes sociales, poder y participación ciudadana. Revista Austral de Ciencias Sociales(26), 23-48.

Carrillo, J. (Marzo de 2018). Revista NEO. Obtenido de https://www.revistaneo.com/articles/2018/05/22 /que-automotriz-lidera-las-redes-sociales

Fuentes, A. (2011). Administración UNAM. Obtenido de http://fcaenlinea.unam.mx/2006/1130/docs/unid ad4.pdf

IDC. (12 de Noviembre de 2018). IDC. Obtenido de https://idconline.mx/corporativo/2018/11/12/par a-que-necesitan-la-nube-las-empresas

Miralles, R. (2010). Cloud computing y protección de datos. IDP. Revista de Internet, Derecho y Política, 14-23. .

NEXU. (2018). NEXU. Obtenido de https://www.nexu.mx/blog/como-ha-influidointernet-en-la-industria-automotriz/
Pérez, G. (2011). La Web 2.0 y la sociedad de la información. Revista Mexicana de Ciencias Políticas y Sociales, 61(212), 57-68.

Riquelme, R. (Noviembre de 2018). El Economista. Obtenido de https://www.eleconomista.com.mx/tecnologia/L a-mitad-de-las-empresas-en-Mexico-estanhaciendo-inversiones-en-la-nube-201806280042.html

Saavedra, M., \& Tapia, B. (2013). El uso de las tecnologías de información y comunicación TIC en las micro, pequeñas y medianas empresas (MIPyME) industriales mexicanas. Enl@ce: Revista Mexicana de Información, Tecnología y Conocimiento.

Vázquez, S. (2015). Tecnologías de almacenamiento de información en el ambiente digital. Revista e-Ciencias de la Información, 5(2), 1-18.

Sampieri, R. (2016). Metodología de la investigación (Sexta ed.). Ciudad de México: McGRAW-HILL.

Martínez, A. (17 de Noviembre de 2018). El Economísta. Obtenido de https://www.eleconomista.com.mx/tecnologia/7 -graficos-sobre-los-usuarios-de-internet-enMexico-en-2018-20180517-0077.html

Copp, E., \& Adame, A. (7 de Diciembre de 2018). Hootsuite. Obtenido de https://blog.hootsuite.com/es/tendencias-enredes-sociales/ 